\title{
Review Article \\ Future Directions and Molecular Basis of Ventilator Associated Pneumonia
}

\author{
Kubra Aykac, Yasemin Ozsurekci, and Sevgen Tanir Basaranoglu \\ Department of Pediatric Infectious Diseases, Hacettepe University Faculty of Medicine, Ankara, Turkey \\ Correspondence should be addressed to Kubra Aykac; kubraklnc.kk@gmail.com
}

Received 31 January 2017; Accepted 14 September 2017; Published 15 October 2017

Academic Editor: Inmaculada Alfageme

Copyright (C) 2017 Kubra Aykac et al. This is an open access article distributed under the Creative Commons Attribution License, which permits unrestricted use, distribution, and reproduction in any medium, provided the original work is properly cited.

\begin{abstract}
Mechanical ventilation is a lifesaving treatment and has complications such as ventilator associated pneumonia (VAP) that lead to high morbidity and mortality. Moreover VAP is the second most common hospital-acquired infection in pediatric intensive care units. Although it is still not well understood, understanding molecular pathogenesis is essential for preventing and treating pneumonia. A lot of microbes are detected as a causative agent of VAP. The most common isolated VAP pathogens in pediatric patients are Staphylococcus aureus, Pseudomonas aeruginosa, and other gram negative bacteria. All of the bacteria have different pathogenesis due to their different virulence factors and host reactions. This review article focused on mechanisms of VAP with molecular pathogenesis of the causative bacteria one by one from the literature. We hope that we know more about molecular pathogenesis of VAP and we can investigate and focus on the management of the disease in near future.
\end{abstract}

\section{Introduction}

1.1. Ventilator Associated Pneumonia. Mechanical ventilation is an essential, lifesaving therapy for patients with critical illness and respiratory failure [1]. These patients are at high risk for complications such as ventilator associated pneumonia (VAP) with a prevalence ranging from $6.6 \%$ to $32 \%[2,3]$. Additionally VAP is a significant problem among pediatric intensive care units due to the fact that it is the second most common hospital-acquired infection after bloodstream infections [4]. Moreover it causes an increase in morbidity, mortality, and length of stay in the hospital, particularly in intensive care unit as well as care costs $[3,5]$.

1.2. Methods. We evaluated both reviews and original articles about molecular basis of ventilator associated pneumonia, new diagnostic biomarkers, and its future directions from database of PUBMED (1986 to 2016). The keywords "molecular" or "ventilator associated pneumonia" or "bacterial pneumonia" or "biomarkers" were used. Last definitions, especially, new biomarkers, and mostly seen bacterial agents were investigated and revealed about ventilator associated pneumonia.
1.3. Definition and Diagnosis of VAP. There is no gold standard, valid definition, and diagnosis for VAP and even the most widely used VAP criteria and definitions are neither sensitive nor specific [1]. In 2016, Centers for Disease Control and Prevention (CDC) reported a module about the definition of VAP as follows: a pneumonia where the patient is on mechanical ventilation for $>2$ calendar days on the date of event, with day of ventilator placement being day 1 , and the ventilator was in place on the date of event or the day before [6].

Clinical suspicion of VAP in a patient is the initial part of diagnosis.

In addition, according to $\mathrm{CDC}$, the standard diagnostic criteria include [6] the following:

For any pediatric patient, at least one of the following:

(i) Fever $>38^{\circ} \mathrm{C}$ or hypothermia of $<36.5^{\circ} \mathrm{C}$,

(ii) Leukopenia $\leq 4000 \mathrm{WBC} / \mathrm{mm}^{3}$ or leukocytosis $\geq$ $15,000 \mathrm{WBC} / \mathrm{mm}^{3}$,

And at least two of the following:

(i) New onset of purulent sputum or change in character of sputum or increased respiratory secretions or increased suctioning requirements, 
(ii) New onset or worsening cough or dyspnea or tachypnea,

(iii) Rales or bronchial breath sounds,

(iv) Worsening gas exchange (e. g., $\mathrm{O}_{2}$ desaturations [e.g., $\left.\mathrm{PaO}_{2} / \mathrm{FiO}^{2} \leq 240\right]$, increased oxygen requirements, or increased ventilator demand),

At least three (only for child $>1$ year old or $\leq 12$ years old) of the following:

(i) Fever of $>38^{\circ} \mathrm{C}$ or hypothermia of $<36.5^{\circ} \mathrm{C}$,

(ii) Leukopenia $\leq 4000 \mathrm{WBC} / \mathrm{mm}^{3}$ or leukocytosis $\geq$ $15,000 \mathrm{WBC} / \mathrm{mm}^{3}$,

(iii) New onset of purulent sputum or change in character of sputum or increased respiratory secretions or increased suctioning requirements,

(iv) New onset or worsening cough or dyspnea, apnea, or tachypnea,

(v) Rales or bronchial breath sounds,

(vi) Worsening gas exchange (e. g., $\mathrm{O}_{2}$ desaturations [e.g., pulse oximetry $<94 \%$ ], increased oxygen requirements, or increased ventilator demand),

At least three (only for infants $\leq 1$ year old) of the following:

(i) Temperature instability,

(ii) Leukopenia $\leq 4000 \mathrm{WBC} / \mathrm{mm}^{3}$ or leukocytosis $\geq$ $15,000 \mathrm{WBC} / \mathrm{mm}^{3}$ and left shift ( $\geq 10 \%$ band forms),

(iii) New onset of purulent sputum or change in character of sputum or increased respiratory secretions or increased suctioning requirements,

(iv) Apnea, tachypnea, and nasal flaring with retraction of chest wall of nasal flaring with grunting,

(v) Wheezing, rales, or rhonchi,

(vi) Cough,

(vii) Bradycardia $(<100$ beats/min) or tachycardia $(>170$ beats/min) [6].

The presence of at least one of the following on one or more (in patients with underlying diseases two or more) serial chest radiographs, new or progressive radiographic infiltrates, consolidation, cavitation, and pneumatoceles in an infant $\leq 1$ year old will also be needed for the diagnosis [6].

Besides physical examination, culture is important because it can establish the causative organism and guide the treatment. Culture specimens can be obtained by tracheal aspirate or bronchoalveolar lavage [4]. However more studies should be performed to achieve the most reasonable culture results [7].

Current studies tend towards to biological markers in the diagnostic algorithm of VAP. It was reported that soluble triggering receptor expressed on myeloid cells- 1 and surfactant protein-D level of bronchoalveolar lavage fluid and serum procalcitonin levels might be useful predictors for VAP [810]. There are a plenty of studies about the risk factors of
VAP. Reintubation, presence of tracheostomy, enteral feeding, prolonged PICU, or hospital stay regardless of illness severity, genetic syndrome, transport out of the PICU, positive blood culture, prior antibiotic usage, bronchoscopy, immunodeficiency, immunosuppressant drugs, neuromuscular blockade, gastric aspiration, mechanic ventilation longer than 3 days, the use of acid-suppressive therapy, neuromuscular diseases, histamine-2 receptor blockers, vasoactive drugs, and presence of a nasoenteral tube were reported to be related to increased risk for VAP $[2,11-16]$.

\section{Molecular Pathogenesis of Pneumonia}

Understanding molecular pathogenesis of pneumonia is essential to prevent and treat pneumonia; however it is still not well understood. Nowadays the studies showed that uncontrollable epithelial cell death is fundamental to the pathogenesis of pneumonia especially in early stages of inflammation. In an animal study, Zou et al. discovered expression of a protein named the mortality factor 4 like 1 (Morf 4l1) increases in humans with pneumonia and it is related to host cell death during pulmonary inflammation [17]. Cytokine response, neutrophil activity, and responsiveness to cytokines and neutrophil lifespan are important in lung infection pathogenesis. The degree of neutrophil activation, generation of reactive oxygen species, and the release of granule proteins are significant in microbial pathogen clearance [18]. Cytotoxins such as $\alpha$-hemolysin (HIa) are also very important in the pathogenesis. HIa is highly potent in lysing bronchial and alveolar epithelial cells, macrophages, and lymphocytes and it is official in proinflammatory processes [19].

\section{Pathogens}

A lot of microbes were detected as a causative agent of VAP. The most common isolated VAP pathogens in pediatric patients are Staphylococcus aureus, Pseudomonas aeruginosa, and other gram negative bacteria [3, 7]. Nonetheless, most of the tracheal isolates from patients with VAP were polymicrobial [7]. All of these pathogens cause VAP by using patients' weakened lung defence systems, resulting from pulmonary and systemic illness and medical therapy, worsening the normal host microbial flora by illness, antibiotics, and mechanic ventilation devices [20].

Initially we should use an antimicrobial therapy which covers the bacteria confirmed by the culture for the treatment rather than broad spectrum antibiotics because of avoiding of antimicrobial resistance of VAP [4]. Antimicrobial-coated endotracheal tubes (EET) are still an investigating target due to the fact that bacteria create biofilm on ETTs and then enter the lungs and cause pneumonia. There are several studies on EETs [21-23]. In 2015 Tokmaji et al. published a review about silver-coated EETs for prevention of VAP and stated limited evidence in reduction of the VAP risk [21]. It is showed by using scanning electron microscopy that gardine- and gendine-coated ETT inhibited the formation of biofilms due to bacteria and were found to be more effective in preventing biofilm growth than the silver ETTs. Nevertheless, several 
animal and clinical studies are needed to validate the efficacy and safety [23].

3.1. Pseudomonas aeruginosa. Secondary nosocomial pneumoniae is one of the frequent causes of deaths in hospital setting in spite of the developing medicine [24] and one of the major causative bacterial pathogens is Pseudomonas aeruginosa with an extremely high mortality rate, particularly in patients with impaired immunity as an opportunistic pathogen $[25,26]$. Therefore, there is an increasing interest in the use of immunoadjuvant therapy to recruit host immunity in patients with such kinds of infections. IL-17 stimulates proliferation of cells in the lymphoid lineage and has crucial roles for survival, development, and homeostasis of lymphocytes [27-29]. IL-17, its T receptor pathway, and TNF- $\alpha$ also have important roles in pulmonary clearance of gram negative bacteria and thereby improving survival [29]. Therefore, recombinant human IL-7 (rhIL-7) is one of the most promising of these new immunoadjuvants and has been effective in decreasing mortality in animals with immunosuppressive state [30].

Shindo et al. [29] demonstrated that rhIL-7 improves survival in secondary $P$. aeruginosa pneumonia. Moreover, they showed the increase in the production of lymphocytes that secrete IFN- $\delta$, IL-17, and TNF- $\alpha$, cytokines that are important in the host defence against both sepsis and pneumoniae caused by $P$. aeruginosa. The increase in those cytokines is important because Pastille et al. [31] stated in an animal model that a disturbed interaction of accessory cells and NK cells may be the cause of the damaged release of IFN- $\delta$ in mice treated with Pseudomonas. Early production of IL-17 seems play a crucial role in infected mice with $P$. aeruginosa despite the fact that the exact role of IL-17 is unclear [32].

Pneumoniae caused by $P$. aeruginosa is a complex process. Some membrane surface elements including flagellum, fimbriae, and polysaccharides are used via a number of aggregated pili to adhere to respiratory epithelia [33, 34]. Immune response of the host is stimulated by means of some virulence factors such as type III secretory protein, quorum-sensing system, and lipopolysaccharides (LPS). Consequently, secreted cytokines, chemotactic factors, and other inflammatory mediators by host cells cause severe lung injury and mortality through activating macrophages, neutrophilic granulocytes, and T cells $[35,36]$. Phosphoinositide 3-kinase (PI3K)/AKT and Ras/Mitogen activated protein kinase (MAPK) have significant roles in the membrane receptors signalling, which are involved in both the inflammatory and immune responses [37,38]. LPS activates protein kinase$\mathrm{C}$ (PKC) and initiates proinflammatory signals such as focal adhesion kinase (FAK), a protein tyrosine kinase, and Ras, a GTPase. PI3K and MAPK activated by FAK and Ras activate ERK and p38 to regulate the expression of cytokines in the immune and inflammatory responses [39-42]. PI3K and MAPKs may be activated by cytokines besides other growth factors to arrange the proliferation and differentiation of cells and the expression of proinflammatory factors [43]. Hou et al. designed a study using TNF- $\alpha$ induced cultured cells to ascertain the anti-inflammatory effect of Qingfei Xiaoyan Wan, a traditional Chinese medicine formula, in animals with $P$. aeruginosa induced acute lung inflammation. They showed that several ingredients of this drug, arctigenin being the principal one, have potential suppression on the primary intracellular immune response and affect the pathways of PI3K/AKT and Ras/MAPK [44].

3.2. Staphylococcus aureus. S. aureus is one of the most common pathogens associated with VAP in the PICU [45]. In a prospective study, 26 children with VAP were investigated and $S$. aureus was detected in $28.4 \%$ of patients with the highest rate [7]. Generally most strains are methicillinsensitive $S$. aureus, but the prevalence of methicillin-resistant strains is increasing, even in community isolates [20]. There are several factors of being respiratory diseases, such as substantial metabolic capabilities, genetic flexibility, ability to adapt to environmental pressures, and exploitation of the immune responses that are evoked [46].

Virulence factor expressions are controlled by a number of regulatory systems in the genome of $S$. aureus such as accessory gene regulatory (Agr) system. This system regulates the expression of surface proteins (protein A) and secreted toxins (toxic shock syndrome toxin, hemolysin, and staphylokinase) [47]. Agr and its regulation are necessary for invasive pulmonary infection. The expression of Agr is required for intracellular life to escape from endosomes. S. aureus can live for long time in neutrophils and in some cases divide within dying cells; thus, this can contribute to a systemic dissemination of the organisms. Due to these features, development of vaccines seems unlikely to be successful. $\delta$ and $\beta$ toxin act to facilitate staphylococcal escape from endosomes in airway epithelial cells [46]. Additionally the lung with increased airway permeability, inhibition of ciliary beat frequency, and neutrophilic response are associated with $\beta$-toxin, that is, a sphingomyelinase which targets the membranes of host cells [48-50]. Similarly $\alpha$-toxin is essential for the pathogenesis of $S$. aureus pneumonia. Qiu et al. studied on isoalantolactone a natural compound from Inula helenium (IAL) against $S$. aureus in vitro and reported that IAL can markedly inhibit the expression of $\alpha$-toxin at very low concentrations [51]. In other studies about $\alpha$-toxin, it is stated that capsaicin and silibinin have prevented $\alpha$-toxinmediated alveolar cell injury through animal studies about pneumonia caused by $S$. aureus $[52,53]$.

Panton-valentine leukocidin (PVL) is a toxin encoded by lukF-PV and lukS-PV genes carried on a bacteriophage. PVL causes the apoptosis of neutrophils via caspases 3 and 9 and inflammation and contributes necrotizing pneumonia [46]. New studies about anti-PVL monoclonal antibodies might be useful for early diagnosis and treatment [54]. For diagnosis of $S$. aureus, Linge et al. suggested that one of the antimicrobial protein midkine was detected in sputum from patients suffering from VAP caused by $S$. aureus [55]. Stulik et al. published their data that airway colonization with MSSA strains with high HIa is a predictor of progression to VAP. Moreover HIa is currently being evaluated for using as active and passive immunization in human trials [19]. Recently, Achouiti et al. investigated host response in mice and found that $S$. aureus pneumonia was associated with a strong rise in 
myeloid-related protein 8/14 in bronchoalveolar lavage fluid and lung tissue [56].

These studies support reasonable approach to antivirulence factors as a new antibacterial target for treatment.

3.3. Klebsiella pneumoniae. Klebsiella pneumoniae is the causative agent of a wide range of infections including pneumonia, bacteremia, and sepsis and is the third most common cause of hospital-acquired infections [57]. This bacteria is rapidly acquiring resistance to all known antibiotics, including carbapenems [58]. Multidrug resistant $K$. Pneumoniae exhibits about $50 \%$ of mortality rate in patients with bloodstream infections $[59,60]$. K. pneumoniae has acquired carbapenemases, which are enzymes capable of breaking down most $\beta$-lactams, including $K$. Pneumoniae (KPCs), carbapenemases of the oxacillinase-48 (OXA-48), and New Delhi Metallo- $\beta$-lactamase (NDM) carbapenemases have important roles in the rapid dissemination of the disease [61]. Various class carbapenemases have been identified worldwide in K. pneumoniae as well as hospitalacquired multiresistant K. Pneumoniae [62, 63].

$K$. pneumoniae siderophores are big contributing factors in the inflammation and bacterial dissemination of the patients with lung infection caused by $K$. pneumoniae according to data of Holden et al. [64]. Additionally, they also showed that master transcription factor hypoxia inducible factor- $1 \alpha$ (HIF- $1 \alpha$ ) is needed for bacterial dissemination in lung epithelial cells. Siderophores, high-affinity iron chelating molecules, have important role for bacterial growth and replication in many gram negative bacteria including $K$. pneumonia [65-67]. Although siderophore-associated iron regulation during bacterial infections is relatively unclear, iron chelation via siderophores induces cytokine secretion of interleukin-8 (IL-8), IL-6, and chemokine (C-C motif) ligand 20 from lung epithelial cells [64, 67]. Siderophores stabilize HIF-1 $\alpha$ in vitro $[68,69]$. HIF-1 $\alpha$ regulates the function of many genes that have some critical roles in glycolysis, inflammation, and angiogenesis [70]. HIF-1 $\alpha$ activation has been associated with innate immunity against infections [71].

The invasive nature of Klebsiella may be attributed to K. pneumonia strain, expressing the $\mathrm{K} 1$ or $\mathrm{K} 2$ capsular antigen [72]. K1/K2 serotype $K$. pneumonia strains are highly pathogenic because of the presence of mucoviscosityassociated gene a (mag A), regulator mucoid phenotype A gene (rmpA), and capsular antigens K1, K2. Those factors induce resistance to phagocytosis through neutrophils and macrophages that are involved in the early innate immune response to intrapulmonary K. pneumonia infection $[72,73]$.

CD36, a scavenger receptor that has a role in the innate immune response to $K$. pneumonia and recognizes pathogen and modified self-ligands, is a host determinant of K. pneumonia pathogenicity and mainly expressed via some cell types including macrophages, endothelial cells, and epithelial cells [74]. Olonisakin et al. [75] showed the critical role of CD36 for optimal control of $K$. pneumonia in the lungs and extrapulmonary bacterial dissemination by mediating recognition of LPS, enhancing alveolar macrophage activity, and managing the optimal cytokine production in the lungs in an acute bacterial pneumonia model. Additionally, CD36 also regulates TLR4/TLR6 complex formation to potentiate NF-kB activity [76].

Chemokines, secreted locally or in paracrine and autocrine fashions by leucocytes and tissue cells, aside from chemokine receptors are crucial therapeutic targets for the diseases [77]. CXC chemokines (alpha chemokines) are subdivided according to presence of the Glu-LeuArg (ELR) tripeptide motif. The ELR-CXC family of chemokines regulates neutrophil recruitment and CXCL8 (interleukin (IL8)); the prototypical ELR-CXC chemokine is commonly detected in infections caused by Klebsiella [78]. Lungs endothelial cells primarily secrete ELR-CXC chemokines and other inflammatory mediators following bacterial activation. A human CXCL8 analogue (G31P), antagonizes both CXCL1 and CXCL2, seems a promising agent in some models such as aspiration pneumoniae and ischemia-reperfusion injury $[79,80]$.

3.4. Acinetobacter baumannii. A. baumannii is increasingly becoming one of the most common pathogens causing VAP [81]. There are very few studies about genetic molecular basis of A. Baumannii infections. Elhosseiny et al. investigated universal stress protein A (UspA) in A. baumannii pneumonia in animals. They highlighted the role of UspA as an important contributor to the A. baumannii virulence and it could be a new therapeutic target [82]. In another animal study phospholipase $\mathrm{D}$ seems to be an A. baumannii virulence factor [83]. In 2015, Méndez et al. suggested that ex vivo proteome of A. baumannii is an important step for diagnostic biomarkers, novel drug targets, and potential vaccine candidates against $A$. baumannii pneumonia [84].

Recently, a connection between host-mediated metal starvation and metabolic stress in A. baumannii pneumonia was reported in a new study published about antimicrobial activity of calprotectin [85]. Moreover, a tumor suppressor protein recently described as immunoregulatory protein Fus1 has a role in the immune response to A. baumannii and Hood et al. stated that this could be a new avenue for immune modulating therapeutic targets [86]. In 2016, it was reported that immunization with an outer membrane nuclease (NucAb) decreased bacterial load, cytokines, and inflammation in mice lungs and, thus, it could be a vaccine candidate in A. baumannii infection [22]. Nowadays, treating multidrug resistance of $A$. baumannii with currently available drugs is difficult; that is, we should start investigating vaccines besides new drugs.

3.5. Escherichia coli. E. coli is the main Enterobacteriaceae caused VAP [87]. Due to increasing multidrug resistance among E. coli, scientists investigate genotypic and phenotypic characteristics of E. coli and aim to discover immunotherapy and vaccine against $E$. coli [88]. Dufour et al. studied the effect of bacteriophage treatment on mice and reported that phage therapy could be a promising therapeutic strategy for VAP [89].

The clearance of microbes from respiratory tract requires systemic and localized inflammatory response controlled by host-derived cytokines [90]. Alveolar epithelial STAT3 activated by IL- 6 family members functions to promote 
neutrophil recruitment and limits infection and injury during E.coli pneumonia [91]. Therefore, Cui et al. tested the proinflammatory effects of TGF- $\beta 1$ in E. coli pneumonia and stated that TGF- $\beta 1$ was associated with improved microbial clearance in rat models of pneumonia while overall survival was not significantly improved [92]. In addition, it was shown that deficiency of tissue-expressed CD47 (integrin associated protein) protects the lung parenchyma whereas deficiency of CD44 (cell-surface receptor for hyaluronic acid) leads to lung injury in E. coli pneumonia in mice $[93,94]$. In 2010, an endogenous mediator called resolvin E1 was presented for the first candidate as a novel therapeutic for acute lung injury and pneumonia due to $E$. coli with an animal study [95]. There are not many studies about molecular activities of Enterobacter spp. Kostiushko and Markelova investigated the cytokine profiles at the experimental Enterobacter pneumonia and reported that local levels of cytokines are different from pneumonia caused by E. coli and Enterobacter spp. [96].

\section{Conclusion}

There are continuing rapid advances in our understanding of the basic mechanisms of VAP. Understanding of these mechanisms may guide the discovery of the possible therapeutic targets for improving host defence, preventing lung injury and infection. Because of increasing antimicrobial resistance and scarcity of new antibiotic discovery there should be more studies about mechanisms of VAP for finding new approaches for prevention and treatment.

\section{Conflicts of Interest}

The authors declare that there are no conflicts of interest regarding the publication of this paper.

\section{References}

[1] 2017, http://www.cdc.gov/nhsn/PDFs/pscManual/10-VAE_ FINAL.pdf.

[2] D. M. Kusahara, C. da Cruz Enz, A. F. M. Avelar, M. A. S. Peterlini, M. da Luz, and G. Pedreira, "Risk factors for ventilator-associated pneumonia in infants and children: A crosssectional cohort study," American Journal of Critical Care, vol. 23, no. 6, pp. 469-476, 2014.

[3] M. F. Patria, G. Chidini, L. Ughi et al., "Ventilator-associated pneumonia in an Italian pediatric intensive care unit: A prospective study," World Journal of Pediatrics, vol. 9, no. 4, pp. 365-368, 2013.

[4] I. Chang and A. Schibler, "Ventilator Associated Pneumonia in Children," Paediatric Respiratory Reviews, vol. 20, pp. 10-16, 2016.

[5] R. J. Brilli, K. W. Sparling, M. R. Lake et al., “The business case for preventing ventilator-associated pneumonia in pediatric intensive care unit patients," Joint Commission Journal on Quality and Patient Safety, vol. 34, no. 11, pp. 629-638, 2008.

[6] 2017, http://www.cdc.gov/nhsn/pdfs/pscmanual/6pscvapcurrent.pdf.

[7] E. Foglia, M. D. Meier, and A. Elward, "Ventilator-associated pneumonia in neonatal and pediatric intensive care unit patients," Clinical Microbiology Reviews, vol. 20, no. 3, pp. 409425, 2007.

[8] P. Ramirez, M. A. Garcia, M. Ferrer et al., "Sequential measurements of procalcitonin levels in diagnosing ventilatorassociated pneumonia," European Respiratory Journal, vol. 31, no. 2, pp. 356-362, 2008.

[9] A. S. Said, M. M. Abd-Elaziz, M. M. Farid, M. A. Abd-Elfattah, M. T. Abdel-Monim, and A. Doctor, "Evolution of surfactant protein-D levels in children with ventilator-associated pneumonia," Pediatric Pulmonology, vol. 47, no. 3, pp. 292-299, 2012.

[10] R. İşgüder, G. Ceylan, H. Ağın, G. Gülfidan, Y. Ayhan, and İ. Devrim, "New parameters for childhood ventilator associated pneumonia diagnosis," Pediatric Pulmonology, vol. 52, no. 1, pp. 119-128, 2017.

[11] M. Almuneef, Z. A. Memish, H. H. Balkhy, H. Alalem, and A. Abutaleb, "Ventilator-associated pneumonia in a pediatric intensive care unit in Saudi Arabia: A 30-month prospective surveillance," Infection Control and Hospital Epidemiology, vol. 25, no. 9, pp. 753-758, 2004.

[12] A. M. Elward, D. K. Warren, and V. J. Fraser, "Ventilatorassociated pneumonia in pediatric intensive care unit patients: Risk factors and outcomes," Pediatrics, vol. 109, no. 5, pp. 758 764, 2002.

[13] M. J. Fayon, M. Tucci, J. Lacroix et al., "Nosocomial pneumonia and tracheitis in a pediatric intensive care unit: A prospective study," American Journal of Respiratory and Critical Care Medicine, vol. 155, no. 1, pp. 162-169, 1997.

[14] A. Torres, R. Aznar, J. M. Gatell et al., "Incidence, risk, and prognosis factors of nosocomial pneumonia in mechanically ventilated patients," American Review of Respiratory Disease, vol. 142, no. 3, pp. 523-528, 1990.

[15] B. D. Albert, D. Zurakowski, L. J. Bechard et al., "Enteral Nutrition and Acid-Suppressive Therapy in the PICU: Impact on the Risk of Ventilator-Associated Pneumonia*," Pediatric Critical Care Medicine, vol. 17, no. 10, pp. 924-929, 2016.

[16] P. Balasubramanian and M. S. Tullu, "Study of VentilatorAssociated Pneumonia in a Pediatric Intensive Care Unit," The Indian Journal of Pediatrics, vol. 81, no. 11, pp. 1182-1186, 2014.

[17] C. Zou, J. Li, S. Xiong et al., "Mortality factor 4 like 1 protein mediates epithelial cell death in a mouse model of pneumonia," Science Translational Medicine, vol. 7, no. 311, Article ID aac7793, 2015.

[18] J. Bordon, S. Aliberti, R. Fernandez-Botran et al., "Understanding the roles of cytokines and neutrophil activity and neutrophil apoptosis in the protective versus deleterious inflammatory response in pneumonia," International Journal of Infectious Diseases, vol. 17, no. 2, pp. e76-e83, 2013.

[19] L. Stulik, S. Malafa, J. Hudcova et al., " $\alpha$-hemolysin activity of methicillin-susceptible Staphylococcus aureus predicts ventilator-associated pneumonia," American Journal of Respiratory and Critical Care Medicine, vol. 190, no. 10, pp. 1139-1148, 2014.

[20] D. R. Park, "The microbiology of ventilator-associated pneumonia," Respiratory Care, vol. 6, pp. 742-763, 2005.

[21] G. Tokmaji, H. Vermeulen, M. C. A. Müller, P. H. S. Kwakman, M. J. Schultz, and S. A. J. Zaat, "Silver-coated endotracheal tubes for prevention of ventilator-associated pneumonia in critically ill patients," Cochrane Database of Systematic Reviews, vol. 12, Article ID CD009201, 2015. 
[22] N. Garg, R. Singh, G. Shukla, N. Capalash, and P. Sharma, "Immunoprotective potential of in silico predicted Acinetobacter baumannii outer membrane nuclease, NucAb," International Journal of Medical Microbiology, vol. 306, no. 1, pp. 1-9, 2016.

[23] I. I. Raad, J. A. Mohamed, R. A. Reitzel et al., "The prevention of biofilm colonization by multidrug-resistant pathogens that cause ventilator-associated pneumonia with antimicrobialcoated endotracheal tubes," Biomaterials, vol. 32, no. 11, pp. 2689-2694, 2011.

[24] American Thoracic Society and Infectious Diseases Society of America, "Guidelines for the management of adults with hospital-acquired, ventilator-associated, and healthcareassociated pneumonia," American Journal of Respiratory and Critical Care Medicine, vol. 171, no. 4, pp. 388-416, 2005.

[25] B. J. Williams, J. Dehnbostel, and T. S. Blackwell, "Pseudomonas aeruginosa: Host defence in lung diseases," Respirology, vol. 15, no. 7, pp. 1037-1056, 2010.

[26] G. F. Sonnenberg and D. Artis, "Innate lymphoid cells in the initiation, regulation and resolution of inflammation," Nature Medicine, vol. 21, no. 7, pp. 698-708, 2015.

[27] C. L. MacKall, T. J. Fry, and R. E. Gress, "Harnessing the biology of IL-7 for therapeutic application," Nature Reviews Immunology, vol. 11, no. 5, pp. 330-342, 2011.

[28] S. A. Corfe and C. J. Paige, "The many roles of IL-7 in B cell development; mediator of survival, proliferation and differentiation," Seminars in Immunology, vol. 24, no. 3, pp. 198-208, 2012.

[29] Y. Shindo, A. G. Fuchs, C. G. Davis et al., "Interleukin 7 immunotherapy improves host immunity and survival in a two-hit model of," Journal of Leukocyte Biology, vol. 101, no. 2, pp. 543554, 2017.

[30] J. Unsinger, C.-A. D. Burnham, J. McDonough et al., "Interleukin-7 ameliorates immune dysfunction and improves survival in a 2-hit model of fungal sepsis," The Journal of Infectious Diseases, vol. 206, no. 4, pp. 606-616, 2012.

[31] E. Pastille, S. Pohlmann, F. Wirsdörfer, A. Reib, and S. B. Flohé, "A disturbed interaction with accessory cells upon opportunistic infection with Pseudomonas aeruginosa contributes to an impaired IFN- $\gamma$ production of NK cells in the lung during sepsis-induced immunosuppression," Journal of Innate Immunity, vol. 21, no. 2, pp. 115-126, 2015.

[32] J. Liu, Y. Feng, K. Yang et al., "Early production of IL-17 protects against acute pulmonary Pseudomonas aeruginosa infection in mice," FEMS Immunology \& Medical Microbiology, vol. 61, no. 2, pp. 179-188, 2011.

[33] C. K. Stover, X. Q. Pham, A. L. Erwin et al., "Complete genome sequence of Pseudomonas aeruginosa PAO1, an opportunistic pathogen," Nature, vol. 406, no. 6799, pp. 959-964, 2000.

[34] S. L. Gellatly and R. E. W. Hancock, "Pseudomonas aeruginosa: new insights into pathogenesis and host defenses," Pathogens and Disease, vol. 67, no. 3, pp. 159-173, 2013.

[35] J. A. Driscoll, S. L. Brody, and M. H. Kollef, “The epidemiology, pathogenesis and treatment of Pseudomonas aeruginosa infections," Drugs, vol. 67, no. 3, pp. 351-368, 2007.

[36] R. T. Sadikot, T. S. Blackwell, J. W. Christman, and A. S. Prince, "Pathogen-host interactions in pseudomonas aeruginosa pneumonia," American Journal of Respiratory and Critical Care Medicine, vol. 171, no. 11, pp. 1209-1223, 2005.

[37] J. M. Kyriakis and J. Avruch, "Mammalian mitogen-activated protein kinase signal transduction pathways activated by stress and inflammation," Physiological Reviews, vol. 81, no. 2, pp. 807$869,2001$.
[38] Y. Zhang, L.-O. Cardell, L. Edvinsson, and C.-B. Xu, "MAPK/ NF- $\kappa$ B-dependent upregulation of kinin receptors mediates airway hyperreactivity: a new perspective for the treatment," Pharmacological Research, vol. 71, pp. 9-18, 2013.

[39] M. T. Diaz-Meco and J. Moscat, "The atypical PKCs in inflammation: NF- $\kappa$ B and beyond," Immunological Reviews, vol. 246, no. 1, pp. 154-167, 2012.

[40] D. S. Johnson and Y. H. Chen, "Ras family of small GTPases in immunity and inflammation," Current Opinion in Pharmacology, vol. 12, no. 4, pp. 458-463, 2012.

[41] M. D. Schaller, "Biochemical signals and biological responses elicited by the focal adhesion kinase," Biochimica et Biophysica Acta (BBA) - Molecular Cell Research, vol. 1540, no. 1, pp. 1-21, 2001.

[42] D. D. Schlaepfer, S. Hou, S.-T. Lim et al., "Tumor necrosis factor- $\alpha$ stimulates focal adhesion kinase activity required for mitogen-activated kinase-associated interleukin 6 expression," The Journal of Biological Chemistry, vol. 282, no. 24, pp. 1745017459, 2007.

[43] A. Tripathi and A. Sodhi, "Growth hormone-induced production of cytokines in murine peritoneal macrophages in vitro: Role of JAK/STAT, PI3K, PKC and MAP kinases," Immunobiology, vol. 214, no. 6, pp. 430-440, 2009.

[44] Y. Hou, Y. Nie, B. Cheng et al., "Qingfei Xiaoyan Wan, a traditional Chinese medicine formula, ameliorates Pseudomonas aeruginosa-induced acute lung inflammation by regulation of PI3K/AKT and Ras/MAPK pathways," Acta Pharmaceutica Sinica B (APSB), vol. 6, no. 3, pp. 212-221, 2016.

[45] V. B. Cooper and C. Haut, "Preventing ventilator- associated pneumonia in children: An evidence- based protocol," Critical Care Nurse, vol. 33, no. 3, pp. 21-29, 2013.

[46] D. Parker and A. Prince, "Immunopathogenesis of Staphylococcus aureus pulmonary infection," Seminars in Immunopathology, vol. 34, no. 2, pp. 281-297, 2012.

[47] P. Recsei, B. Kreiswirth, M. O’Reilly, P. Schlievert, A. Gruss, and R. P. Novick, "Regulation of exoprotein gene expression in Staphylococcus aureus by agr," MGG Molecular \& General Genetics, vol. 202, no. 1, pp. 58-61, 1986.

[48] F. M. Aarestrup, H. D. Larsen, N. H. R. Eriksen, C. S. Elsberg, and N. E. Jensen, "Frequency of $\alpha$ - and $\beta$-haemolysin in Staphylococcus aureus of bovine and human origin. A comparison between pheno- and genotype and variation in phenotypic expression," APMIS-Acta Pathologica, Microbiologica et Immunologica Scandinavica, vol. 107, no. 4, pp. 425-430, 1999.

[49] C. S. Kim, S.-Y. Jeon, Y.-G. Min et al., "Effects of $\beta$-toxin of Staphylococcus aureus on ciliary activity of nasal epithelial cells," The Laryngoscope, vol. 110, no. 12, pp. 2085-2088, 2000.

[50] A. Hayashida, A. H. Bartlett, T. J. Foster, and P. W. Park, "Staphylococcus aureus beta-toxin induces lung injury through syndecan-1," The American Journal of Pathology, vol. 174, no. 2, pp. 509-518, 2009.

[51] J. Qiu, X. Niu, J. Wang et al., "Capsaicin protects mice from community-associated methicillin-resistant Staphylococcus aureus pneumonia," PLoS ONE, vol. 7, no. 3, Article ID e33032, 2012.

[52] X. Wang, J. Dong, X. Dai et al., "Silibinin in vitro protects A549 cells from staphylococcus aureus-mediated injury and in vivo alleviates the lung injury of staphylococcal pneumonia," Planta Medica, vol. 79, no. 2, pp. 110-115, 2013.

[53] J. Qiu, M. Luo, J. Wang et al., "Isoalantolactone protects against Staphylococcus aureus pneumonia," FEMS Microbiology Letters, vol. 324, no. 2, pp. 147-155, 2011. 
[54] C. E. Okolie, A. Cockayne, C. Penfold, and R. James, "Engineering of the LukS-PV and LukF-PV subunits of Staphylococcus aureus Panton-Valentine leukocidin for Diagnostic and Therapeutic Applications," BMC Biotechnology, vol. 13, article no. 103, 2013.

[55] H. M. Linge, C. Andersson, S. L. Nordin et al., "Midkine is expressed and differentially processed during chronic obstructive pulmonary disease exacerbations and ventilator-associated pneumonia associated with staphylococcus aureus infection," Molecular Medicine, vol. 19, no. 1, pp. 314-323, 2013.

[56] A. Achouiti, T. Vogl, A. J. Van Der Meer et al., "Myeloid-related protein-14 deficiency promotes inflammation in staphylococcal pneumonia," European Respiratory Journal, vol. 46, no. 2, pp. 464-473, 2015.

[57] S. S. Magill, J. R. Edwards, W. Bamberg et al., "Multistate pointprevalence survey of health care-associated infections," The New England Journal of Medicine, vol. 370, no. 13, pp. 1198-1208, 2014.

[58] D. M. Sievert, P. Ricks, J. R. Edwards et al., "Antimicrobialresistant pathogens associated with healthcare- associated infections: summary of data reported to the national healthcare safety network at the centers for disease control and prevention, 2009-2010," Infection Control and Hospital Epidemiology, vol. 34, no. 1, pp. 1-14, 2013.

[59] L. S. Munoz-Price, L. Poirel, R. A. Bonomo et al., "Clinical epidemiology of the global expansion of Klebsiella pneumoniae carbapenemases," The Lancet Infectious Diseases, vol. 13, no. 9, pp. 785-796, 2013.

[60] M. Tumbarello, P. Viale, C. Viscoli et al., "Predictors of mortality in bloodstream infections caused by Klebsiella pneumoniae carbapenemase-producing K. pneumoniae: importance of combination therapy," Clinical Infectious Diseases, vol. 55, no. 7, pp. 943-950, 2012.

[61] C.-R. Lee, J. H. Lee, K. S. Park, Y. B. Kim, B. C. Jeong, and S. H. Lee, "Global dissemination of carbapenemase-producing Klebsiella pneumoniae: Epidemiology, genetic context, treatment options, and detection methods," Frontiers in Microbiology, vol. 7, article no. 895, 2016.

[62] T. Tängdén and C. G. Giske, "Global dissemination of extensively drug-resistant carbapenemase-producing Enterobacteriaceae: clinical perspectives on detection, treatment and infection control," Journal of Internal Medicine, vol. 277, no. 5, pp. 501-512, 2015.

[63] P. Nordmann, T. Naas, and L. Poirel, "Global spread of carbapenemase producing Enterobacteriaceae," Emerging Infectious Diseases, vol. 17, no. 10, pp. 1791-1798, 2011.

[64] V. I. Holden, P. Breen, S. Houle, C. M. Dozois, and M. A. Bachman, "Klebsiella pneumoniae siderophores induce inflammation, bacterial dissemination, and HIF-1 $\alpha$ stabilization during pneumonia," mBio, vol. 7, no. 5, Article ID e01397-16, 2016.

[65] M. S. Lawlor, C. O'Connor, and V. L. Miller, "Yersiniabactin is a virulence factor for Klebsiella pneumoniae during pulmonary infection," Infection and Immunity, vol. 75, no. 3, pp. 1463-1472, 2007.

[66] M. A. Bachman, P. Breen, V. Deornellas et al., "Genome-wide identification of Klebsiella pneumoniae fitness genes during lung infection," mBio, vol. 6, no. 3, 2015.

[67] V. I. Holden and M. A. Bachman, "Diverging roles of bacterial siderophores during infection," Metallomics, vol. 7, no. 6, pp. 986-995, 2015.

[68] V. I. Holden, S. Lenio, R. Kuick, S. K. Ramakrishnan, Y. M. Shah, and M. A. Bachman, "Bacterial siderophores that evade or overwhelm lipocalin 2 induce hypoxia inducible factor $1 \alpha$ and proinflammatory cytokine secretion in cultured respiratory epithelial cells," Infection and Immunity, vol. 82, no. 9, pp. 38263836, 2014.

[69] A. L. Nelson, A. J. Ratner, J. Barasch, and J. N. Weiser, "Interleukin-8 secretion in response to aferric enterobactin is potentiated by siderocalin," Infection and Immunity, vol. 75, no. 6, pp. 3160-3168, 2007.

[70] A. Palazon, A. Goldrath, V. Nizet, and R. Johnson, "HIF transcription factors, inflammation, and immunity," Immunity, vol. 41, no. 4, pp. 518-528, 2014.

[71] N. V. Kirienko, D. R. Kirienko, J. Larkins-Ford, C. Wählby, G. Ruvkun, and F. M. Ausubel, "Pseudomonas aeruginosa disrupts Caenorhabditis elegans iron homeostasis, causing a hypoxic response and death," Cell Host \& Microbe, vol. 13, no. 4, pp. 406416, 2013.

[72] L. K. Siu, K. M. Yeh, J. C. Lin, C. P. Fung, and F. Y. Chang, "Klebsiella pneumoniae liver abscess: a new invasive syndrome," The Lancet Infectious Diseases, vol. 12, no. 11, pp. 881-885, 2012.

[73] V. L. Yu, D. S. Hansen, C. K. Wen et al., "Virulence characteristics of Klebsiella and clinical manifestations of K. pneumoniae bloodstream infections," Emerging Infectious Diseases, vol. 13, no. 7, pp. 986-993, 2007.

[74] J. Canton, D. Neculai, and S. Grinstein, "Scavenger receptors in homeostasis and immunity," Nature Reviews Immunology, vol. 13, no. 9, pp. 621-634, 2013.

[75] T. F. Olonisakin, H. Li, Z. Xiong et al., "CD36 provides host protection against klebsiella pneumoniae intrapulmonary infection by enhancing lipopolysaccharide responsiveness and macrophage phagocytosis," The Journal of Infectious Diseases, vol. 214, no. 12, pp. 1865-1875, 2016.

[76] C. R. Stewart, L. M. Stuart, K. Wilkinson et al., "CD36 ligands promote sterile inflammation through assembly of a Toll-like receptor 4 and 6 heterodimer," Nature Immunology, vol. 11, no. 2, pp. 155-161, 2010.

[77] J. Wei, J. Peng, B. Wang et al., "CXCR1/CXCR2 antagonism is effective in pulmonary defense against Klebsiella pneumoniae infection," BioMed Research International, vol. 2013, Article ID 720975, 6 pages, 2013.

[78] B. B. Aggarwal, S. Shishodia, S. K. Sandur, M. K. Pandey, and G. Sethi, "Inflammation and cancer: how hot is the link?" Biochemical Pharmacology, vol. 72, no. 11, pp. 1605-1621, 2006.

[79] X. Zhao, J. R. Town, F. Li, W. Li, X. Zhang, and J. R. Gordon, "Blockade of neutrophil responses in aspiration pneumonia via ELR-CXC chemokine antagonism does not predispose to airway bacterial outgrowth," Pulmonary Pharmacology and Therapeutics, vol. 23, no. 1, pp. 22-28, 2010.

[80] X. Zhao, J. R. Town, A. Yang et al., "A novel ELR-CXC chemokine antagonist reduces intestinal ischemia reperfusioninduced mortality, and local and remote organ injury," Journal of Surgical Research, vol. 162, no. 2, pp. 264-273, 2010.

[81] B. A. Almomani, A. McCullough, R. Gharaibeh, S. Samrah, and F. Mahasneh, "Incidence and predictors of 14-day mortality in multidrug-resistant Acinetobacter baumannii in ventilatorassociated pneumonia," The Journal of Infection in Developing Countries, vol. 9, no. 12, pp. 1323-1330, 2015.

[82] N. M. Elhosseiny, M. A. Amin, A. S. Yassin, and A. S. Attia, "Acinetobacter baumannii universal stress protein A plays a pivotal role in stress response and is essential for pneumonia and sepsis pathogenesis," International Journal of Medical Microbiology, vol. 305, no. 1, pp. 114-123, 2015. 
[83] A. C. Jacobs, I. Hood, K. L. Boyd et al., "Inactivation of phospholipase D diminishes Acinetobacter baumannii pathogenesis," Infection and Immunity, vol. 78, no. 5, pp. 1952-1962, 2010.

[84] J. A. Méndez, J. Mateos, A. Beceiro et al., "Quantitative proteomic analysis of host-pathogen interactions: a study of Acinetobacter baumannii responses to host airways," BMC Genomics, vol. 16, no. 1, article no. 422, 2015.

[85] L. J. Juttukonda, W. J. Chazin, and E. P. Skaar, "Acinetobacter baumannii coordinates urea metabolism with metal import to resist host-mediated metal limitation," mBio, vol. 7, no. 5, Article ID e01475-16, 2016.

[86] M. I. Hood, R. Uzhachenko, K. Boyd, E. P. Skaar, and A. V. Ivanova, "Loss of mitochondrial protein Fusl augments host resistance to Acinetobacter baumannii infection," Infection and Immunity, vol. 81, no. 12, pp. 4461-4469, 2013.

[87] J. Chastre and J. Fagon, "Ventilator-associated pneumonia," American Journal of Respiratory and Critical Care Medicine, vol. 165, no. 7, pp. 867-903, 2002.

[88] J. Messika, F. Magdoud, O. Clermont et al., "Pathophysiology of Escherichia coli ventilator-associated pneumonia: Implication of highly virulent extraintestinal pathogenic strains," Intensive Care Medicine, vol. 38, no. 12, pp. 2007-2016, 2012.

[89] N. Dufour, L. Debarbieux, M. Fromentin, and J.-D. Ricard, "Treatment of Highly Virulent Extraintestinal Pathogenic Escherichia coli Pneumonia with Bacteriophages," Critical Care Medicine, vol. 43, no. 6, pp. e190-e198, 2015.

[90] http://www.medscape.com/viewarticle/717400.

[91] L. J. Quinton, M. R. Jones, B. E. Robson, B. T. Simms, J. A. Whitsett, and J. P. Mizgerd, "Alveolar epithelial STAT3, IL-6 family cytokines, and host defense during Escherichia coli pneumonia," American Journal of Respiratory Cell and Molecular Biology, vol. 38, no. 6, pp. 699-706, 2008.

[92] X. Cui, F. Zeni, Y. Vodovitz et al., "TGF- $\beta 1$ increases microbial clearance but worsens lung injury during Escherichia coli pneumonia in rats," Cytokine, vol. 24, no. 4, pp. 115-127, 2003.

[93] X. Su, M. Johansen, M. R. Looney, E. J. Brown, and M. A. Matthay, "CD47 deficiency protects mice from lipopolysaccharide-induced acute lung injury and Escherichia coli pneumonia," The Journal of Immunology, vol. 180, no. 10, pp. 6947-6953, 2008.

[94] Q. Wang, P. Teder, N. P. Judd, P. W. Noble, and C. M. Doerschuk, "CD44 deficiency leads to enhanced neutrophil migration and lung injury in Escherichia coli pneumonia in mice," The American Journal of Pathology, vol. 161, no. 6, pp. 2219-2228, 2002.

[95] H. Seki, K. Fukunaga, M. Arita et al., “The anti-inflammatory and proresolving mediator resolvin E1 protects mice from bacterial pneumonia and acute lung injury," The Journal of Immunology, vol. 184, no. 2, pp. 836-843, 2010.

[96] A. V. Kostiushko and E. V. Markelova, "The cytokines profiles at the enterobacter pneumonia during experiment," Pathologicheskaia Fiziologiia i Eksperimental'naia Terapiia, vol. 4, pp. 27-30, 2010 . 


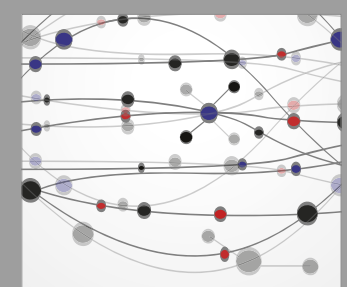

The Scientific World Journal
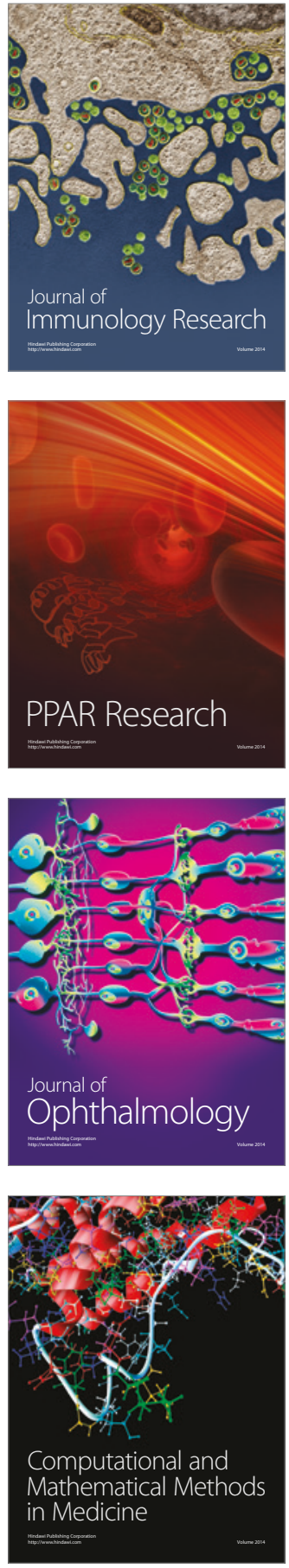

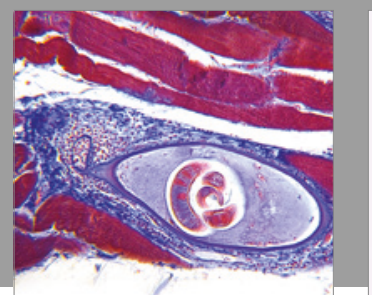

Gastroenterology Research and Practice
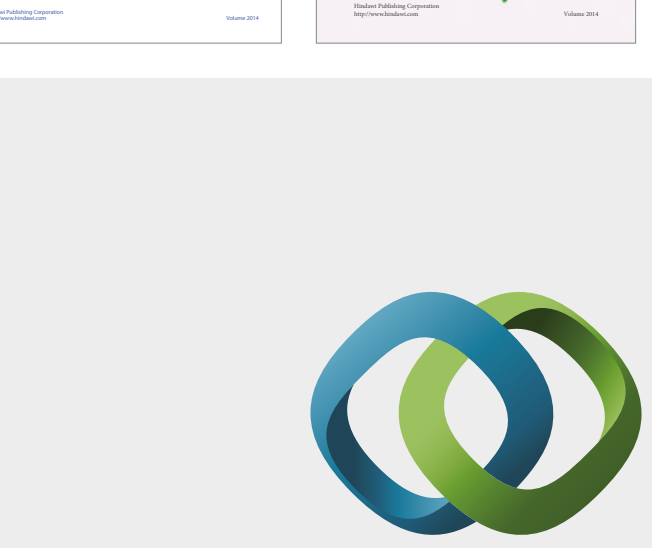

\section{Hindawi}

Submit your manuscripts at

https://www.hindawi.com
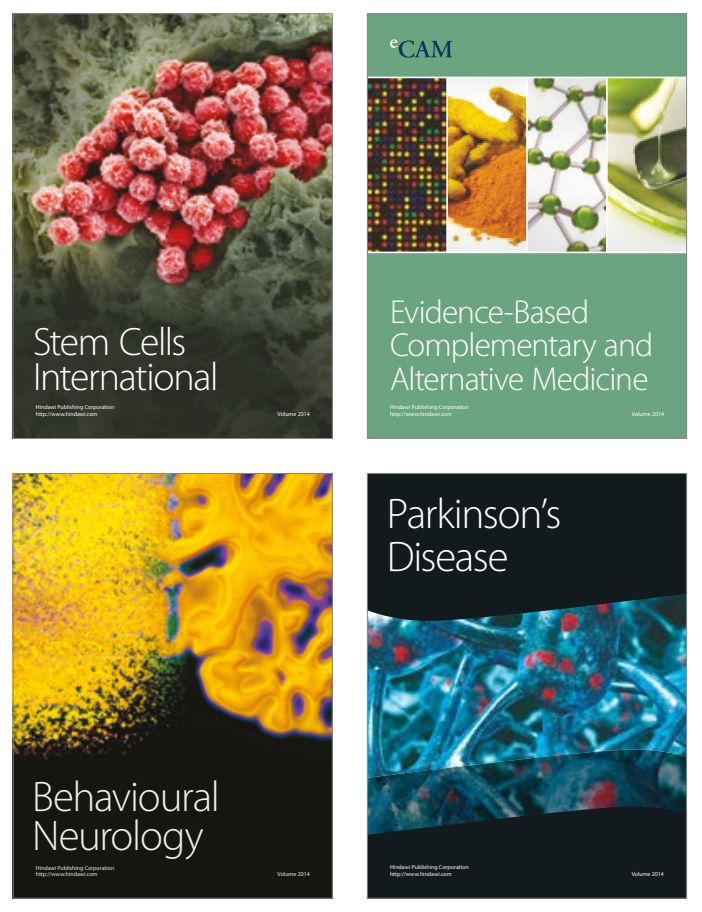
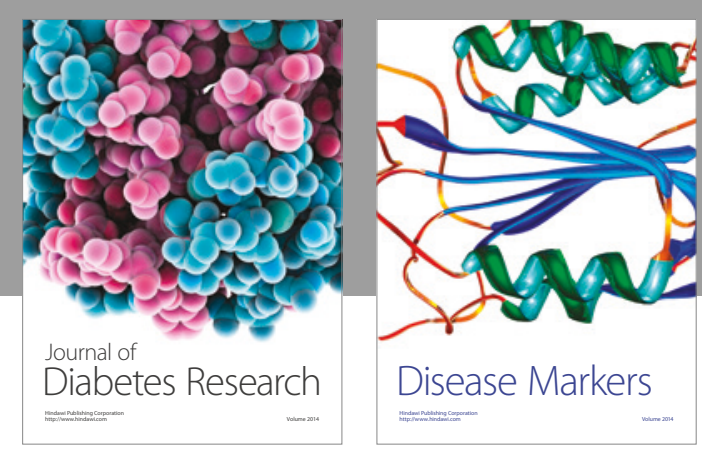

Disease Markers
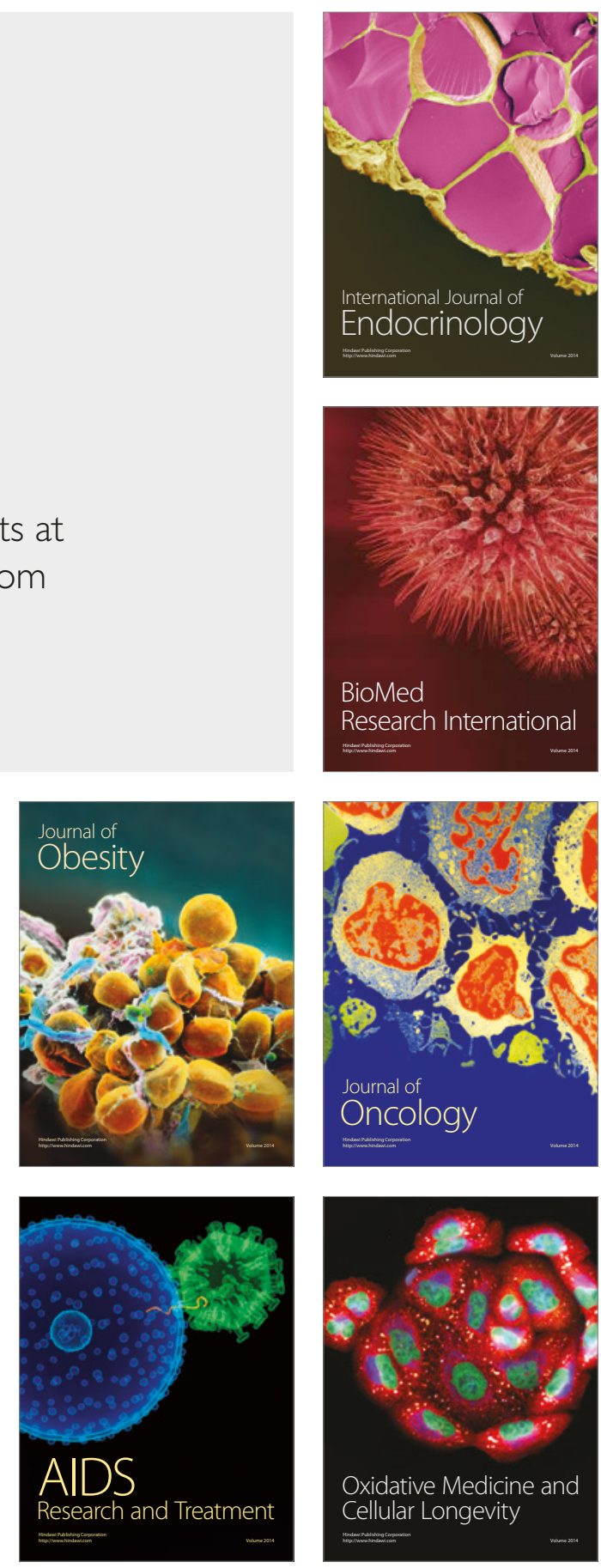